\title{
Fusion of NUP214 to ABL1 on amplified episomes in T-cell acute lymphoblastic leukemia
}

\author{
C. Graux $^{1,2,13}$, J. Cools ${ }^{1,3,13}$, C. Melotte ${ }^{1}$, H. Quentmeier ${ }^{4}$, A. Ferrando ${ }^{5}$, R. Levine ${ }^{5}$, J.R. Vermeesch ${ }^{1}$, M. Stul ${ }^{1}$, \\ B. Dutta ${ }^{6}$, N. Boeckx ${ }^{2}$, A. Bosly ${ }^{7}$, P. Heimann ${ }^{8}$, A. Uyttebroeck ${ }^{9}$, N. Mentens ${ }^{1,3}$, R. Somers ${ }^{1,3}$, R.A.F. MacLeod ${ }^{4}$, \\ H.G. Drexler ${ }^{4}$, A.T. Look $^{5}$, D.G. Gilliland ${ }^{5,10,11}$, L. Michaux ${ }^{12}$, P. Vandenberghe ${ }^{1,2}$, I. Wlodarska ${ }^{1}$,
} Peter Marynen $^{1,3} \&$ Anne Hagemeijer ${ }^{1}$

In T-cell acute lymphoblastic leukemia (T-ALL), transcription factors are known to be deregulated by chromosomal translocations, but mutations in protein tyrosine kinases have only rarely been identified ${ }^{1-3}$. Here we describe the extrachromosomal (episomal) ${ }^{4}$ amplification of $A B L 1$ in 5 of $90(5.6 \%)$ individuals with T-ALL, an aberration that is not detectable by conventional cytogenetics. Molecular analyses delineated the amplicon as a 500-kb region from chromosome band 9q34, containing the oncogenes ABL1 and NUP214 (refs. $5,6)$. We identified a previously undescribed mechanism for activation of tyrosine kinases in cancer: the formation of episomes resulting in a fusion between NUP214 and ABL1. We detected the NUP214-ABL1 transcript in five individuals with the $A B L 1$ amplification, in 5 of $85(5.8 \%)$ additional individuals with T-ALL and in 3 of 22 T-ALL cell lines. The constitutively phosphorylated tyrosine kinase NUP214-ABL1 is sensitive to the tyrosine kinase inhibitor imatinib ${ }^{7,8}$. The

recurrent cryptic NUP214-ABL1 rearrangement is associated with increased $H O X$ expression ${ }^{1}$ and deletion of $C D K N 2 A^{9}$, consistent with a multistep pathogenesis of T-ALL. NUP214$A B L 1$ expression defines a new subgroup of individuals with T-ALL who could benefit from treatment with imatinib.

The Philadelphia translocation, resulting in the BCR-ABL1 fusion gene, is typically found in chronic myeloid leukemia (CML) and precursor B-cell acute lymphoblastic leukemia (B-ALL) but is exceptionally rare in T-ALL ${ }^{2,5,10}$. To study the potential involvement of $A B L 1$ rearrangements in T-ALL, we screened 90 cases by fluorescence in situ hybridization (FISH), using $B C R$ and $A B L 1$ probes. We observed no $B C R-A B L 1$ fusion signals, confirming the low frequency of this rearrangement in T-ALL. But we observed marked amplifica- tion (more than ten signals per nucleus) of $A B L 1$ in 5 of 90 individuals with T-ALL (Fig. 1a and Tables 1 and 2). Notably, the additional $A B L 1$ signals were extrachromosomal. Extrachromosomal amplification of oncogenes has been observed on double minute (dmin) chromosomes ${ }^{11}$, visible by standard cytogenetics, or on cytogenetically invisible units, called episomes ${ }^{4}$. In the cases we studied, no dmin chromosomes were visible by G or R banding (Supplementary Fig. 1 online), suggesting that $A B L 1$ amplification occurred on episomes.

Detailed FISH mapping of the episomes confirmed that they contained ABL1, LAMC3 and NUP214 (also called CAN), three genes localized within a 500-kb region on chromosome region 9q34 (Fig. 1a,c). Probes for ASS, FUBP3 and VAV2 did not hybridize to the episomes (Fig. 1a,c). The $5^{\prime}$ end of $A B L 1$ could not be detected on the episomes in four of six cases, delineating the proximal breakpoint in the first intron of $A B L 1$. Cohybridization of the $3^{\prime} A B L 1$ and NUP214 probes on the episomes confirmed the presence of both genes on the same episomes (Supplementary Fig. 1 online). Microarray-based comparative genomic hybridization (array CGH) confirmed the amplification of ABL1 and LAMC3 in the four cases that could be analyzed (Fig. 1b,c). BAC-57C19 sequences (5' ABL1) were amplified only in individual 1, confirming the FISH findings (Fig. $\mathbf{1 b}$ and Table 1). BAC-5N16 sequences were not amplified in any of the four individuals, further delimiting the amplified region to a maximal size of $1 \mathrm{Mb}$ (Fig. 1b,c). Array CGH also showed deletion of BAC-149I2, containing the tumor-suppressor gene CDKN2A (also called p16), in individuals 2-4, an observation that was confirmed by FISH (Fig. 1a,b and Table 2). In individuals 1-3, amplification of ABL1 was confirmed by Southern blotting (Supplementary Fig. 2 online).

The selective absence of the $5^{\prime}$ end of ABL1 in the amplicon suggested that $A B L 1$ might be involved in the generation of a fusion gene. We therefore carried out RACE-PCR on ABL1 transcripts from

\footnotetext{
${ }^{1}$ Department of Human Genetics and ${ }^{2}$ Department of Hematology, University of Leuven, Leuven, Belgium. ${ }^{3}$ Department of Human Genetics, Flanders Interuniversity Institute for Biotechnology, Leuven, Belgium. ${ }^{4}$ German Collection of Microorganisms and Cell Cultures, Braunschweig, Germany. ${ }^{5}$ Dana-Farber Cancer Institute, Harvard Medical School, Boston, Massachusetts, USA. ${ }^{6}$ Microarray Facility, Flanders Interuniversity Institute for Biotechnology, Leuven, Belgium. ${ }^{7}$ Department of Hematology, Cliniques universitaires UCL de Mont-Godinne, Yvoir, Brussels, Belgium. ${ }^{8}$ Department of Medical Genetics, Erasme Hospital, Free University of Brussels, Brussels, Belgium. ${ }^{9}$ Department of Pediatric Hemato-oncology, University of Leuven, Leuven, Belgium. ${ }^{10}$ Brigham and Women's Hospital and ${ }^{11}$ Howard Hughes Medical Institute, Harvard Medical School, Boston, Massachusetts, USA. ${ }^{12}$ St. Luc, Brussels, Belgium. ${ }^{13}$ These authors contributed equally to this work. Correspondence should be addressed to P.M. (peter.marynen@med.kuleuven.ac.be) or A.H. (anne.hagemeijer@med.kuleuven.ac.be).
} 

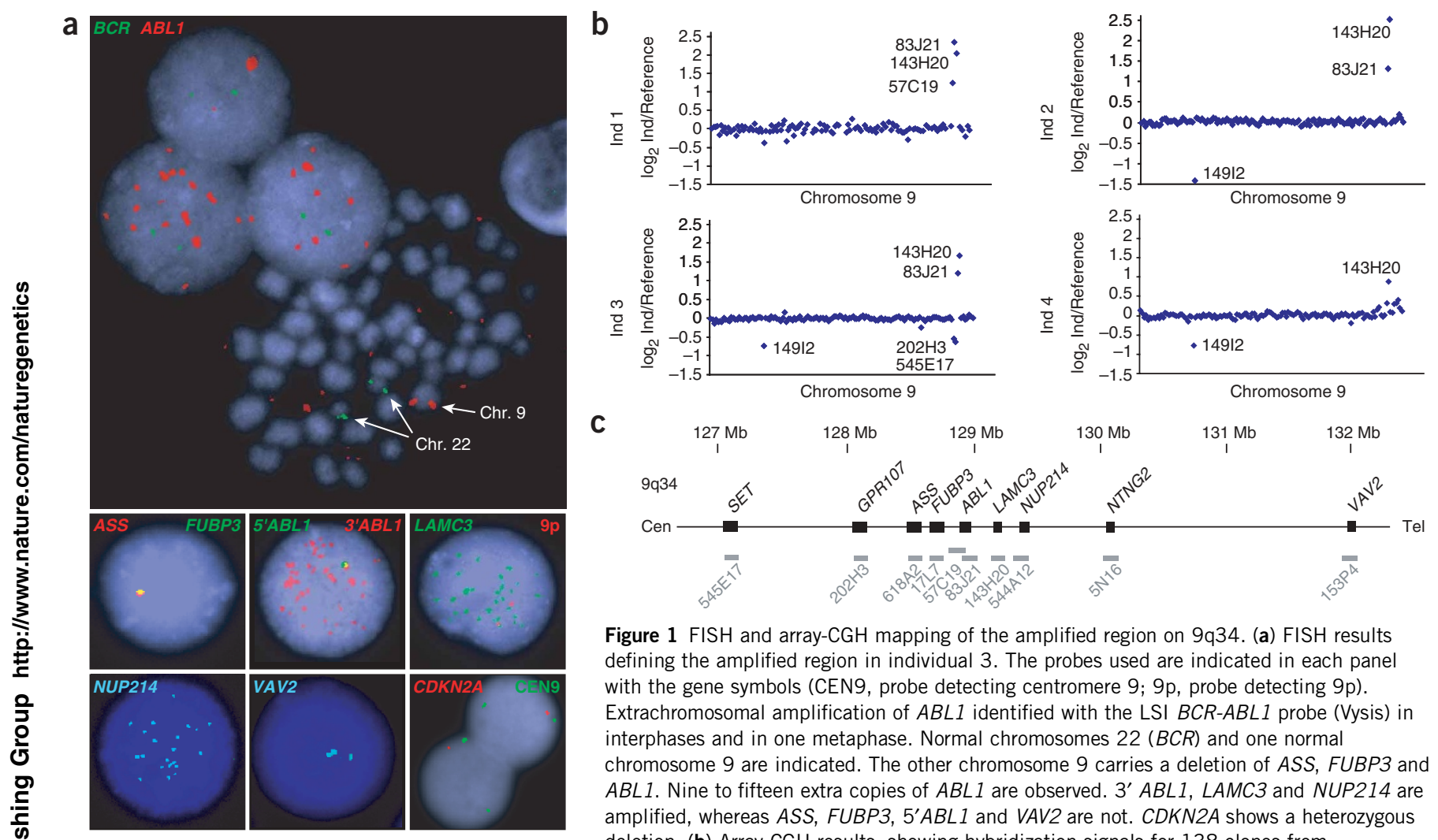

C

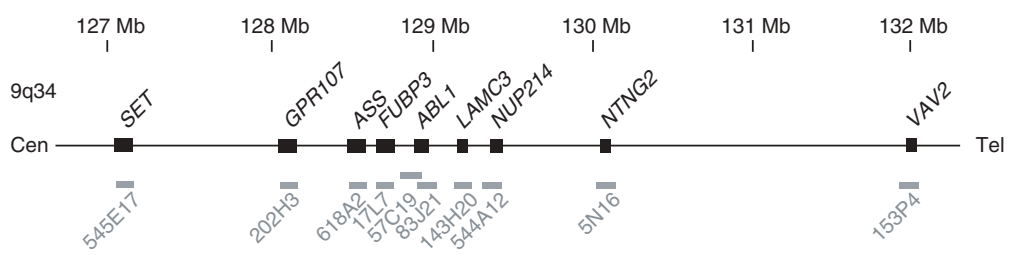

Figure $1 \mathrm{FISH}$ and array-CGH mapping of the amplified region on 9q34. (a) FISH results defining the amplified region in individual 3 . The probes used are indicated in each panel with the gene symbols (CEN9, probe detecting centromere 9; 9p, probe detecting 9p).

Extrachromosomal amplification of $A B L 1$ identified with the LSI BCR-ABL1 probe (Vysis) in interphases and in one metaphase. Normal chromosomes $22(B C R)$ and one normal chromosome 9 are indicated. The other chromosome 9 carries a deletion of ASS, FUBP3 and $A B L 1$. Nine to fifteen extra copies of $A B L 1$ are observed. 3' ABL1, LAMC3 and NUP214 are amplified, whereas ASS, FUBP3, 5'ABL1 and VAV2 are not. CDKN2A shows a heterozygous deletion. (b) Array-CGH results, showing hybridization signals for 138 clones from chromosome 9, confirm the amplification of ABL1 and LAMC3. NTNG2 is not amplified and $5^{\prime} A B L 1$ is amplified only in individual 1 . The array does not contain a probe for the NUP214 locus. Clone 14912 contains CDKN2A and CDKN2B. (c) Locations of the probes used for FISH and array CGH along chromosome 9. Black boxes represent genes, gray boxes represent BACs. Cen, centromeric side; Tel, telomeric side.

individual 4. Sequencing of the PCR products identified an in-frame fusion between exon 31 of NUP214 and exon 2 of ABL1. Subsequent RT-PCR confirmed the presence of NUP214-ABL1 fusion transcripts in five individuals with $A B L 1$ amplification from whom cDNA was available (Fig. 2). These results are compatible with a model in which the genomic region from $A B L 1$ to NUP214 is circularized, generating a NUP214-ABL1 fusion gene (Fig. 3a), and the copy number of the episome is increased owing to unequal segregation during cell division. Consistent with this model, we observed a variable number of episomes (5-50) in different cells from a single individual. In individual 3, episome formation may have originated from a deletion, as one chromosome 9 carries a deletion of a region slightly larger than the amplified region (Fig. 1a,b and Table 1). Similar deletions associated with amplification of MYC on dmin chromosomes or episomes have been reported ${ }^{12}$.

To confirm these findings, we screened an additional 85 individuals with T-ALL by RT-PCR. We detected the NUP214-ABL1 fusion transcript in 5 of these individuals (Fig. 2a and Table 2). We next

Table 1 FISH results in six individuals with abnormal $A B L 1$ hybridization patterns

\begin{tabular}{|c|c|c|c|c|c|c|c|c|c|c|c|c|}
\hline \multirow[b]{2}{*}{ Individual } & \multicolumn{2}{|c|}{ LSI $B C R-A B L 1$} & \multicolumn{10}{|c|}{ Number of signals observed } \\
\hline & Nucleia & Metaphases $^{\mathrm{b}}$ & $\begin{array}{l}373 \mathrm{~J} 8 \\
(N / B L)\end{array}$ & $\begin{array}{c}202 \mathrm{H} 3 \\
(\text { GPR107) }\end{array}$ & $\begin{array}{c}618 \mathrm{~A} 2 \\
(\text { ASS })\end{array}$ & $\begin{array}{c}17 L 7 \\
\text { (FUBP3) }\end{array}$ & $\begin{array}{c}57 C 19 \\
\left(5^{\prime} A B L 1\right)\end{array}$ & $\begin{array}{c}83 \mathrm{~J} 21 \\
\left(3^{\prime} A B L 1\right)\end{array}$ & $\begin{array}{l}143 \mathrm{H} 20 \\
(L A M C 3)\end{array}$ & $\begin{array}{c}544 \mathrm{~A} 12 \\
(N \cup P 214)\end{array}$ & $\begin{array}{l}\text { 153P4 } \\
\text { (VAV2) }\end{array}$ & $\begin{array}{l}83 N 9 \\
(L H X 3)\end{array}$ \\
\hline $1(\mathrm{BM})$ & M (82\%) & $3 / 9$ & 2 & 2 & 2 & 2 & $M$ & $M$ & $M$ & $M$ & 2 & 2 \\
\hline $2(\mathrm{~PB})$ & $M(75 \%)$ & $12 / 19$ & 2 & 2 & 2 & 2 & 2 & M & M & M & 2 & 2 \\
\hline $3(\mathrm{BM})$ & $M(76 \%)$ & $14 / 20$ & 2 & 1 & 1 & 1 & 1 & M & M & M & 2 & 2 \\
\hline $4(\mathrm{BM})$ & $M(23 \%)$ & $16 / 40$ & 2 & 2 & 2 & 2 & 2 & M & M & M & $3(20 \%)$ & 2 \\
\hline $5(\mathrm{BM})$ & $M(6 \%)$ & $13 / 30$ & 2 & 2 & 2 & 2 & $\mathrm{M}$ & M & $\mathrm{M}$ & M & 2 & 2 \\
\hline $6(\mathrm{PE})$ & M (96\%) & $9 / 10$ & NA & NA & 2 & 2 & 2 & M & NA & $M$ & 2 & NA \\
\hline $6(\mathrm{BM})$ & M (16\%) & $1 / 1$ & & & & & & & & & & \\
\hline
\end{tabular}

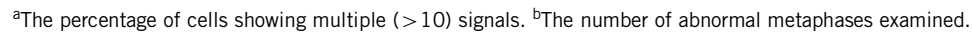

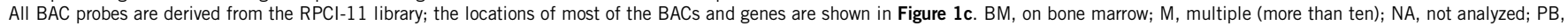
on peripheral blood; $\mathrm{PE}$, on pleural effusion. 
a

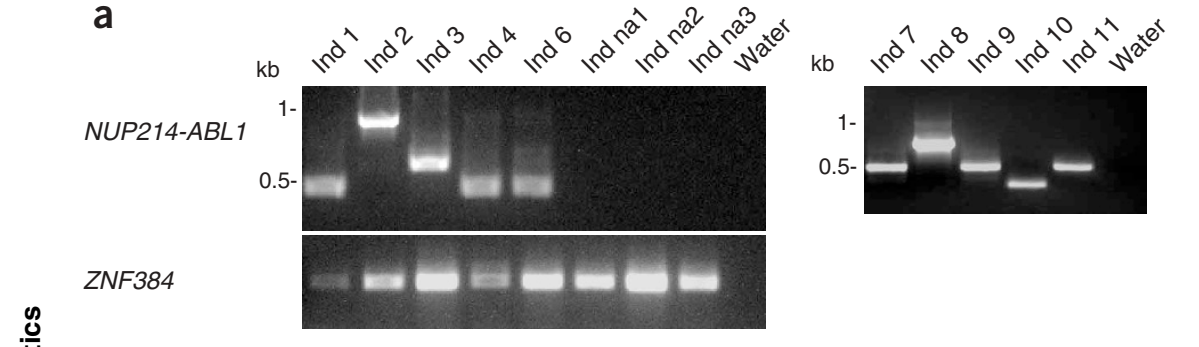

b

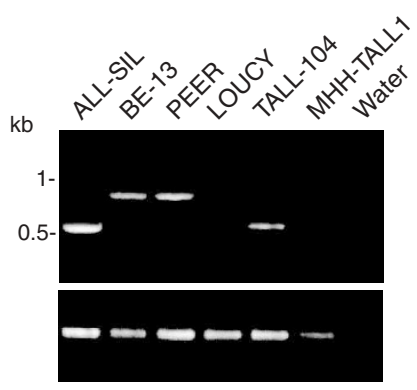

C

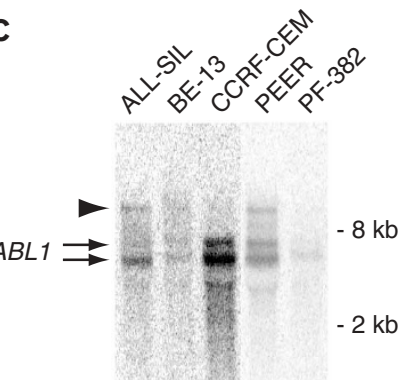

d Ind 2

Ind 10

Ind 1, 4, 6,

$7,9,11$

Ind 3,

ALL-SIL, TALL-104

Ind 8,

PEER, BE-13
NUP214 (exon 23)

ABL1 (exon 2) aggcggcagatggccagtcaggcacCagAAGCCCTTCAGCGGCCAGTAGCATCTGAC

$\begin{array}{lllllllllllllllllll}R & R & Q & M & A & S & Q & A & P & E & A & L & Q & R & P & V & A & S & D\end{array}$

NUP214 (exon 29)

$A B L 1$ (exon 2) tctgggttcagctttgccaagct cagAAGCCCTTCAGCGGCCAGTAGCATCTGAC $\begin{array}{lllllllllllllllllll}S & G & F & S & F & C & Q & A & S & E & A & L & Q & R & P & V & A & S & D\end{array}$

NUP214 (exon 31) $\quad$ ABL1 (exon 2) gggggctt ggatccacagctacctcaaAAGCCCTTCAGCGGCCAGTAGCATCTGAC $\begin{array}{lllllllllllllllllll}G & G & F & G & S & T & A & T & S & K & A & L & Q & R & P & V & A & S & D\end{array}$

NUP214 (exon 32)

$A B L 1$ (exon 2)

ttgggtt tcctctccaaacaaaacagAAGCCCTTCAGCGGCCAGTAGCATCTGAC $\begin{array}{llllllllllllllllllllll}\mathrm{F} & \mathrm{G} & \mathrm{F} & \mathrm{S} & \mathrm{S} & \mathrm{P} & \mathrm{N} & \mathrm{K} & \mathrm{T} & \mathbf{E} & \mathbf{A} & \mathrm{L} & \boldsymbol{Q} & \mathrm{R} & \mathrm{P} & \mathrm{V} & \mathrm{A} & \mathrm{S} & \mathrm{D}\end{array}$

NUP214 (exon 34)

$A B L 1$ (exon 2) ttctctggtttggatcaggcacaggagAAGCCCTTCAGCGGCCAGTAGCATCTGAC

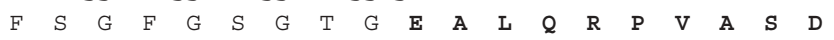

Figure 2 Detection of the NUP214-ABL1 fusion transcript by RT-PCR and northern blotting. (a) Detection of different NUP214-ABL1 fusion transcripts in five individuals with T-ALL with $A B L 1$ amplification (individuals 1-4 and 6), and absence of this fusion in individuals with T-ALL lacking $A B L 1$ amplification (individuals na1-na3). NUP214-ABL1 fusion transcripts were also detected in 5 of 85 additional individuals with T-ALL screened by RT-PCR (individuals 7-11). (b) Detection of different NUP214-ABL1 fusion transcripts in four T-ALL cell lines by RT-PCR. (c) Detection of an aberrant $A B L 1$ transcript (arrowhead) in the cell lines PEER, ALL-SIL and BE-13 by northern blotting. (d) Sequence of the different variant NUP214-ABL1 transcripts detected. All fusions are in-frame (sequence translated with the one-letter amino acid abbreviations).

tested human T-ALL cell lines for the presence of the fusion. We detected the NUP214-ABL1 transcript in 3 of 22 independent T-ALL cell lines: ALL-SIL, PEER and TALL-104, and in BE-13, a tetraploid subline of PEER (Fig. 2b and Supplementary Table 1 online). The presence of an aberrant $A B L 1$ transcript in the cell lines was also confirmed by northern blotting (Fig. 2c). Taken together, these data indicate that $\sim 6 \%$ of individuals with T-ALL carry a cryptic NUP214$A B L 1$ fusion gene.

Among T-ALL cases, we observed variants of the NUP214-ABL1 fusion gene due to different breakpoints in NUP214 (ranging from intron 23 to intron 34; Fig. 2d). All breakpoints in $A B L 1$ occurred in intron 1, and so exon 2 of $A B L 1$ is present in all fusion variants; this coincides with the $A B L 1$ breakpoint observed in Philadelphia-positive CML and B-ALL. The NUP214-ABL1 fusion mRNAs are predicted to encode proteins of 2,210-3,175 amino acids with molecular weights of ح39-333 kDa (Fig. 3b).

NUP214 is a component of the nuclear pore complex, which mediates nucleocytoplasmic transport ${ }^{13}$. NUP214 is widely expressed and is involved in the pathogenesis of acute myeloid leukemia associated with the $\mathrm{t}(6 ; 9)(\mathrm{p} 23 ; \mathrm{q} 34)$ DEK-NUP214 fusion ${ }^{6}$. In the DEK-NUP214 fusion protein, however, the C-terminal region of NUP214 (encoded by exons 18-36) is present, whereas the predicted NUP214-ABL1 fusions retain the N-terminal region of NUP214, which includes the predicted coiled-coil domains that may serve as oligomerization motifs (Fig. 3b).
ABL1 is an ubiquitously expressed cytoplasmic tyrosine kinase that is fused to BCR in CML and precursor B-ALL cases with the $\mathrm{t}(9 ; 22)(\mathrm{q} 34 ; \mathrm{q} 11)$ translocation $^{5,10}$ and to ETV6 in leukemias with the $t(9 ; 12)(q 34 ; p 13)$ translocation ${ }^{14}$. Like BCR-ABL1 and ETV6ABL1 fusion proteins, the NUP214-ABL1 fusions contain the SH3, SH2 and kinase domains of ABL1 (Fig. 3b), suggesting that NUP214ABL1 acts as a constitutively activated tyrosine kinase. We assessed this possibility by analyzing tyrosine phosphorylation of NUP214-ABL1 and CRKL, a direct target of the ABL1 kinase ${ }^{15}$. We detected the NUP214-ABL1 fusion protein in the cell lines PEER, BE-13 and ALLSIL, using antibodies directed against ABL1 or NUP214 (Fig. 4a). NUP214-ABL1 and CRKL were phosphorylated in the cell lines expressing NUP214-ABL1, whereas CRKL was not phosphorylated in the cell line LOUCY, which does not express NUP214-ABL1 (Fig. 4a). Addition of imatinib, a selective inhibitor of ABL1 kinase activity $^{7}$, decreased phosphorylation of both NUP214-ABL1 and CRKL (Fig. 4b) and inhibited the proliferation of ALL-SIL (Fig. 4c). The proliferation of PEER and BE-13 was not affected by imatinib, although the phosphorylation of NUP214-ABL1 was inhibited (Supplementary Fig. 3 online). This is probably due to the fact that the growth of these cell lines became independent of NUP214ABL1 activity because they acquired additional mutations. Similar findings were previously reported in cell lines expressing BCR-ABL1 (ref. 16). Phosphorylation of CRKL was also inhibited by imatinib in primary bone marrow cells from individual 4 (Fig. 4d). These results 
a

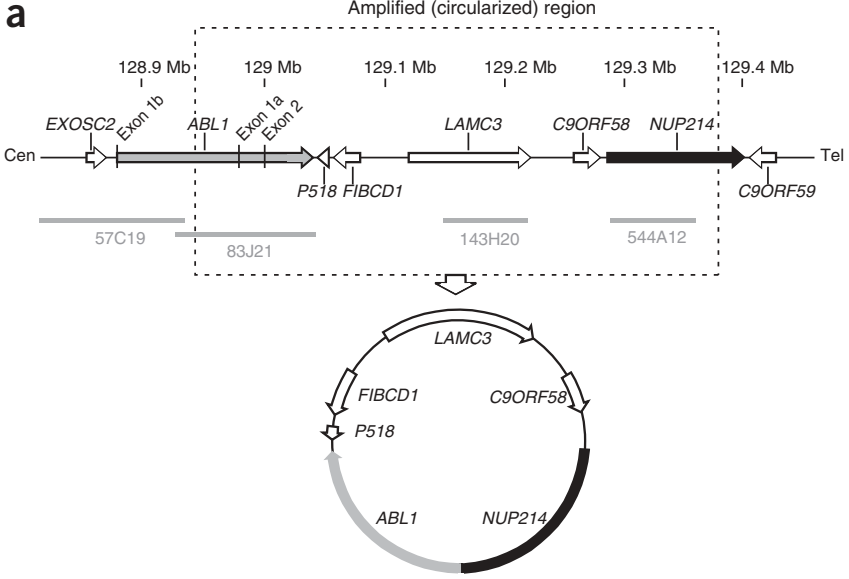

b
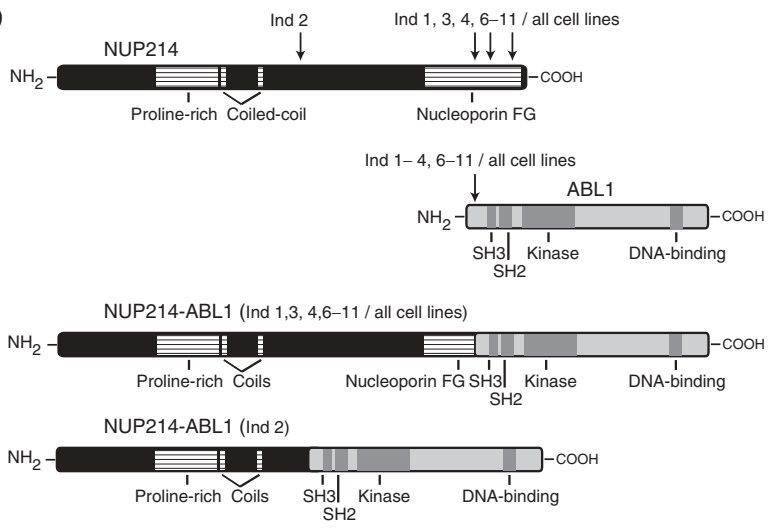

Figure 3 Schematic representation of the amplified region and the NUP214-ABL1 fusion protein. (a) Detailed scheme of the amplified (circularized) region, and the proposed structure of the episome. Genes (arrows), BAC clones (gray bars), and their positions along chromosome 9 are indicated. Cen, centromeric side; Tel, telomeric side. (b) Schematic representation of the NUP214 and ABL1 proteins. Two main NUP214-ABL1 fusion proteins are generated: a shorter fusion (239 kDa) present in only one individual, and longer fusions (310-333 kDa) present in most individuals with T-ALL with ABL1 amplification. All fusions contain the predicted coiled-coil domains of NUP214 and the SH3, SH2 and tyrosine kinase domains of ABL1.

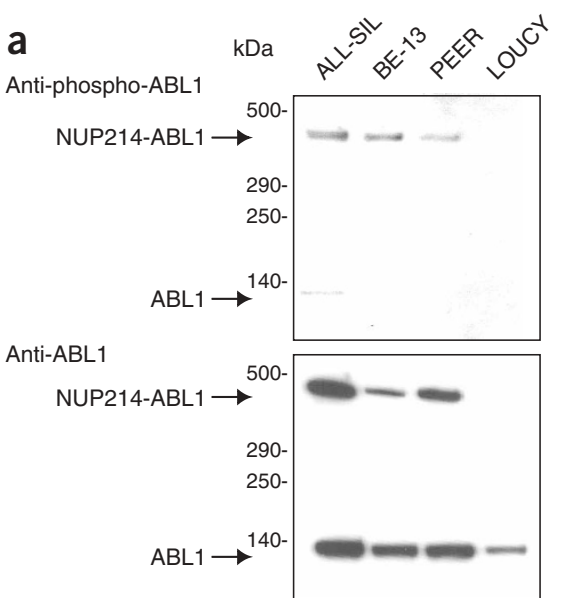

Anti-NUP214

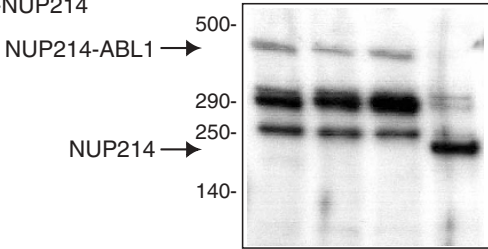

Anti-phospho-CRKL

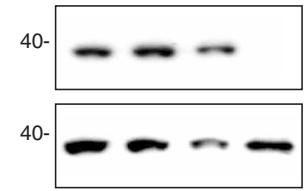

CDKN2A and CDKN2B (also called $p 15$ ) in seven of the nine cases we were able to evaluate. Screening for oncogenes known to be involved in T-ALL identified the mutually exclusive overexpression of TLX1 (also called HOX11) and TLX3 (also called HOX11L2) in four and five individuals, respectively (Table 2). Similar observations were made in the T-ALL cell lines, where expression of TLX1 and NKX2-5 was reported for ALL-SIL and PEER, respectively (Supplementary Table 1 online $)^{18}$. These data provide genetic support for a multi-step b Imatinib $(\mu \mathrm{M})$
Anti-phospho-ABL1
Anti-ABL1
Anti-phospho-CRKL Anti-CRKL

d Imatinib $(\mu \mathrm{M})$ Anti-phospho-CRKL

Anti-CRKL
C

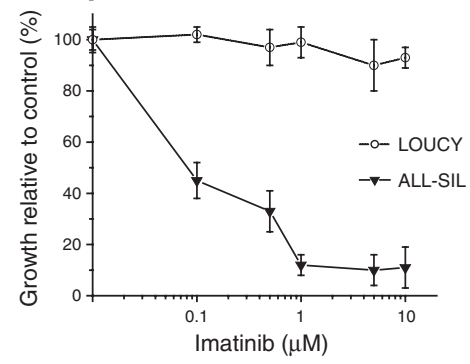

Figure 4 Characterization of the NUP214-ABL1 fusion protein. (a) Using antibodies to ABL1 or NUP214, the NUP214-ABL1 fusion protein could be detected in the cell lines ALL-SIL, BE-13 and PEER, but not in the NUP214-ABL1-negative cell line LOUCY. NUP214-ABL1 was phosphorylated in the three cell lines, and ABL1 was also phosphorylated in ALL-SIL. Phosphorylation of NUP214-ABL1 correlated with phosphorylation of CRKL. (b) Imatinib treatment of ALL-SIL results in a dose-dependent decrease in phosphorylation of NUP214ABL1 and CRKL. (c) Imatinib treatment has a dose-dependent inhibitory effect on the growth of ALL-SIL cell line. As expected, no effect was observed on the LOUCY cell line, also indicating that the inhibitory effect observed with ALL-SIL is not due to a general toxic effect of imatinib on T cells. (d) Treatment of bone marrow cells from individual 4 with imatinib results in decreased phosphorylation of CRKL, indicating an inhibitory effect of imatinib on NUP214$A B L 1$ in leukemic cells from this individual. 
Table 2 Characteristics of 11 individuals with T-ALL with ABL1 amplification or NUP214-ABL1 fusion

\begin{tabular}{|c|c|c|c|c|c|c|c|c|c|c|c|c|}
\hline \multirow[b]{2}{*}{ Individual } & \multirow[b]{2}{*}{ Sex } & \multirow{2}{*}{\multicolumn{2}{|c|}{ Age }} & \multirow[b]{2}{*}{$\begin{array}{l}\text { Percent } \\
\text { blast }^{\mathrm{b}}\end{array}$} & \multirow[b]{2}{*}{$\begin{array}{c}\text { Immune } \\
\text { phenotype }^{c}\end{array}$} & \multirow[b]{2}{*}{ Karyotype $^{d}$} & \multicolumn{2}{|c|}{ FISH results } & \multicolumn{2}{|c|}{ Expression data } & \multirow[b]{2}{*}{$\begin{array}{l}\text { Response to } \\
\text { therapy, outcome }\end{array}$} & \multirow[b]{2}{*}{ OS } \\
\hline & & & & & & & $C D K N 2 A^{f}$ & $A B L 1$ & $\begin{array}{l}\text { NUP214- } \\
\text { ABL1 }\end{array}$ & Otherg & & \\
\hline 1 & M & 52 & 134 & 96 & Cortical & $46, X Y, \operatorname{del}(12)(p 13)[4] / 46, X Y[1]$ & $+/+$ & Amp & + & $T L X 1$ & $\mathrm{CR}$, early relapse & 7 \\
\hline 2 & $M$ & 3 & 162 & 95 & Cortical & $46, X Y[12]$ & $-1-$ & Amp & + & $T L X 1$ & $\mathrm{CR}$ & $57+$ \\
\hline 3 & $\mathrm{M}$ & 23 & 81 & 92 & Cortical & $\begin{array}{c}48, X Y, t(3 ; 11)(p 12 ; p 15), t(7 ; 10)(q 35 ; q 24), t(8 ; 10) \\
(q 21 ; q 21),+11,+12[5] / 46, X Y[4]\end{array}$ & $-/+$ & Amp & + & $T L X 1$ & $\begin{array}{l}\text { CR, alloBMT, } \\
\text { toxic death }\end{array}$ & 10 \\
\hline 4 & $\mathrm{M}$ & 7 & 196 & 88 & Mature & $46, X Y[33]$ & $-1+$ & Amp & + & $T L X 3$ & $\mathrm{CR}$ & $40+$ \\
\hline 5 & M & 6 & 52 & 82 & Pre-T & $47, X Y, \operatorname{del}(6)(q 21),+8[12] / 46, X Y[4]$ & $+/+$ & Amp & NA & NA & CR, early relapse & 14 \\
\hline 6 & M & 25 & 8 & $40^{h}$ & Mature ${ }^{i}$ & $\begin{array}{c}46, Y, \operatorname{add}(X)(p 22), t(8 ; 22)(p 22 ; q 12) \\
\operatorname{del}(13)(q 14 q 22)[10]\end{array}$ & $-1+$ & Amp & + & $T L X 3$ & Early toxic death & 0.5 \\
\hline 7 & $\mathrm{~F}$ & Ped & NA & 96 & Cortical & Near tetraploid with del(11)(q23) & $-/-^{j}$ & NA & + & $T L X 3$ & $\mathrm{CR}$ & $194+$ \\
\hline 8 & $\mathrm{~F}$ & Ped & NA & 86 & Mature ${ }^{\mathrm{i}}$ & NA & $-/-j$ & NA & + & & $\mathrm{CR}$, early relapse & 7 \\
\hline 9 & $\mathrm{M}$ & 9 & NA & NA & NA & NA & NA & NA & + & $T L X 3$ & CR & $14+$ \\
\hline 10 & M & 4 & NA & NA & NA & NA & $-1-j$ & NA & + & $T L X 3$ & CR & $176+$ \\
\hline 11 & $\mathrm{~F}$ & 31 & NA & 75 & Pre-T & $46, X X[20]$ & NA & NA & + & $T L X 1$ & Early toxic death & 1 \\
\hline
\end{tabular}

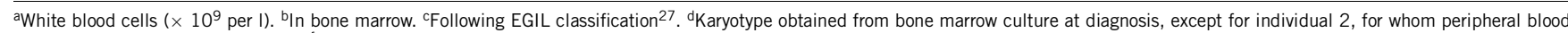
was analyzed. ${ }^{e}$ Overall survival (mo). ${ }^{\dagger}+/+$, no deletion; $-/+$, hemizygous deletion; -/-, homozygous deletion. sMolecular screening for BCR-ABL1, SIL-TAL1, ETV6-RUNX1, TLX1 and TLX3 transcripts and $M L L$ rearrangement (Southern blot). ${ }^{\mathrm{h}} 40 \%$ blasts in bone marrow and $77 \%$ in pleural effusion. i'Mature T phenotype with aberrant expression of CD13. ${ }^{\mathrm{j} R e s u l t s}$ obtained by quantitative PCR (not by FISH).

+ , alive at last analysis; Amp, amplified; CR, complete remission; NA, not available; Ped, pediatric.

pathogenesis of T-ALL: deletion of a tumor-suppressor gene $(C D K N 2 A, C D K N 2 B)$, deregulated expression of a transcription factor (TLX1,TLX3) and expression of a constitutively activated tyrosine kinase (NUP214-ABL1).

Our results identify the episomal fusion of NUP214 to ABL1 as a new mechanism for the generation of a fusion gene. This gene rearrangement is cryptic by conventional cytogenetics but readily detected by FISH using a commercially available $A B L 1$ probe. Amplification of $A B L 1$ in a subset of individuals with T-ALL was recently reported, although the molecular and physiologic consequences of amplification were not elucidated ${ }^{19}$. FISH with the $A B L 1$ probes is distinctive and seems to be pathognomonic for the presence of the NUP214-ABL1 fusion in T-ALL. We observed hybridization of ABL1 on $9 \mathrm{q} 34$, as expected, as well as at multiple extrachromosomal sites in metaphases and multiple signals in most interphase cells. It will be interesting to investigate whether this fusion also occurs in T-ALL as a result of $\mathrm{t}(9 ; 9)(\mathrm{q} 34 ; \mathrm{q} 34)$ or in other hematological malignancies, particularly $B C R-A B L 1$-negative myeloproliferative diseases.

Survival data for the eleven individuals that we studied are indicative of a rather aggressive course of disease in the four adults with the NUP214-ABL1 fusion (two early relapses and two toxic deaths; Table 2). The finding that NUP214-ABL1 is sensitive to the tyrosine kinase inhibitor imatinib suggests that new therapeutic approaches in T-ALL with the NUP214-ABL1 fusion may improve outcome or decrease treatment-related morbidity. This study demonstrates the power of high-resolution array CGH for the detection of acquired genetic unbalances in cancer cells. We show that cryptic (episomal) amplifications may generate new fusion genes, in addition to cryptic deletions ${ }^{20}$. Taken together, these findings suggest that genome-wide screens for deletions and amplifications of tyrosine kinases in a broad spectrum of hematological malignancies and solid tumors may identify more of these aberrations.

\section{METHODS}

Affected individuals. We retrospectively selected 90 individuals with T-ALL from the database of the Department of Human Genetics (Leuven) for the initial FISH screening. We later added an additional individual (individual 6, from the Erasme Hospital, Brussels) to the study. We screened a set of 85 individuals with T-ALL from the database of the Dana-Farber Cancer Institute (Boston) for the presence of the NUP214-ABL1 fusion by RT-PCR. We reviewed the clinical diagnosis, morphology and immunophenotypic data. This study was approved by the Ethical Committee of the Medical Faculty of the University of Leuven and informed consent was obtained from all subjects.

Cytogenetics and FISH. We carried out cytogenetic studies on bone marrow or blood cells using direct or short-term cultures without mitogens and $\mathrm{R}$ banding. Karyotypes were described according to the International System for Human Cytogenetic Nomenclature ${ }^{21}$. In cases where bone marrow was not available, we karyotyped lymph node or pleural effusions. We carried out FISH on stored fixed cell suspension originally used for karyotyping, as described ${ }^{22}$. We were able to hybridize successfully the same metaphases up to three times. Initial screening was done using the LSI BCR-ABL ES (Vysis) translocation probe. On average, we scored ten metaphases and 200 nuclei. We investigated cases showing aberrant hybridization signals using a panel of BAC probes mapping at $9 \mathrm{q} 34$ (Fig. 1c and Table 1). We obtained BACs from the RPCI11 library.

Array CGH. We carried out array CGH using Code Linked Slides (AP Biotech) containing the 3,527 BAC clones from the Wellcome Trust Sanger Institute $1 \mathrm{Mb}$ Clone $\mathrm{Set}^{23}$, a gift from N. P. Carter (The Wellcome Trust Sanger Institute, UK). We amplified BAC DNA by degenerate oligonucleotide-primed $\mathrm{PCR}^{23}$. We spotted aminolinked PCR products at a concentration of $200 \mathrm{ng}$ $\mu \mathrm{l}^{-1}$ on the slides using a Molecular Dynamics Generation III printer (AP Biotech). We printed the clones in two replicates at different positions on the array. We labeled test and reference genomic DNA using the Bioprime DNA Labeling System (Invitrogen) using Cy3- and Cy5-labeled dCTPs (AP Biotech). We prepared probes and preblocked the slide performed as described ${ }^{23}$. We hybridized the slides for $48 \mathrm{~h}$ under coverslips in a humid chamber saturated with $20 \%$ formamide and $2 \times$ saline sodium citrate. We carried out posthybridization washes and image and data analysis as described ${ }^{24}$. Spot intensities were corrected for the local background. Only spots with signal intensities of Cy5 and Cy 3 twofold above background signal intensities were retained. For each clone, we calculated a ratio of $\mathrm{Cy} 5$ over $\mathrm{Cy} 3$ fluorescent intensity. We normalized the data by dividing the fluorescent intensity ratio at each spot by the mean of all ratios of the autosomes. Two values of the duplicate clones were averaged and a $\log _{2}$ value was calculated. If the variation among the two intensity ratios was larger than $10 \%$, the data point was eliminated from the analysis. $\log _{2}$ ratios between -0.2 and 0.2 were accepted to be normal. Ratios 
below or above this normal range were interpreted to be due to a clone deletion or duplication, respectively. If the $\log _{2}$ ratio was above 1 , the clone was considered to be amplified.

Cell culture and western blotting. We incubated bone marrow cells in RPMI1640 medium supplemented with $10 \%$ fetal calf serum for $2 \mathrm{~h}$ in the presence of different concentrations of imatinib. We lysed cells in $1.5 \times$ sample buffer, separated them using SDS-PAGE and transferred them to PVDF membranes. We cultured T-ALL cell lines in RPMI-1640 medium supplemented with 20\% fetal calf serum. For dose-response curves, we grew $3 \times 10^{5}$ cells per $\mathrm{ml}$ in 24-well plates with different concentrations of imatinib and determined the number of viable cells initially and after $48 \mathrm{~h}$ of incubation. We calculated the percentage of viable cells relative to the control (no imatinib) at each time point for three independent wells.

For western blotting, we cultured the cell lines in the presence of different concentrations of imatinib for $2 \mathrm{~h}$, pelleted them and lysed them in cold lysis buffer containing $1 \mathrm{mM} \mathrm{NaVO}_{4}$ and protease inhibitors. We separated the proteins on NuPAGE Tris-Acetate gels (Invitrogen) and transferred them to PVDF membranes. We used antibodies directed to phosphorylated ABL1, ABL1, phosphorylated CRKL and CRKL (Cell Signaling). Peroxidase-labeled antibodies to mouse or rabbit Ig were from AP Biotech. Antibody to NUP214, directed against the C-terminal part of NUP214 (ref. 25), was provided by G. Grosveld (St Jude Children's Research Hospital, Memphis, Tennessee, USA).

PCR. We carried out RACE as described previously ${ }^{26}$. We synthesized cDNA with ABL1-R1 (primer sequences available on request) and then carried out 을 PCR with the adaptor primers ${ }^{26}$ and the nested ABL1 primers ABL1-R2 and ABL1-R3. We then cloned and sequenced the PCR products. We carried out direct RT-PCR to screen for the presence of NUP214-ABL1 transcripts using the primers NUP20 (exon 20), NUP28 (exon 28), NUP29 (exon 29) and NUP31 (exon 31), combined with ABL1-R2 and ABL1-R3. All PCR products were sequenced. As control for RNA quality, we amplified ZNF384 or ABL1 using the primer pairs ZNF384-F and ZNF384-R, and ABL1-FA2 (exon 2) and ABL1-RA3 (exon 3), respectively.

Northern blotting. We separated $10 \mu \mathrm{g}$ of total RNA on a $1 \%$ formaldehyde agarose gel and transferred it to a nylon filter (Gene Screen, DuPont). We hybridized the filter with a random-primed $\mathrm{P}^{32}$-dCTP labeled probe spanning ABL1 exons 2 and 3.

(2) URLs. Locations of genes and probes, and gene structures were determined based on Ensembl data (http://www.ensembl.org). Sequence similarities were Do analyzed using the BLAST algorithm at http://www.ncbi.nlm.nih.gov/BLAST. The BAC clones were obtained from http://bacpac.chori.org.

\section{ACKNOWLEDGMENTS}

This text presents research results of the Belgian program of Interuniversity Poles of attraction initiated by the Belgian State, Prime Minister's Office, Science Policy Programming. The scientific responsibility is assumed by the authors. The authors thank the Mapping Core and Map Finishing groups of the Wellcome Trust Sanger Institute for initial clone supply and verification. This work was supported by grants from the Belgian Federation against Cancer (J.C.), the Fonds voor Wetenschappelijk Onderzoek-Vlaanderen (P.M.) and the National Institutes of Health (A.T.L., D.G.G.). J.C. is a postdoctoral researcher and P.V. is a clinical investigator of the Fonds voor Wetenschappelijk Onderzoek-Vlaanderen. C.G. received a grant from the Belgian Hematological Society and a grant from the Franqui-De Roover Foundation (Salus Sanguinis). D.G.G. is an Investigator of the Howard Hughes Medical Institute and a Doris Duke Distinguished Clinical Scientist.

\section{COMPETING INTERESTS STATEMENT}

The authors declare that they have no competing financial interests.
Received 3 June; accepted 9 August 2004

Published online at http://www.nature.com/naturegenetics/

1. Ferrando, A.A. et al. Gene expression signatures define novel oncogenic pathways in $T$ cell acute lymphoblastic leukemia. Cancer Cell 1, 75-87 (2002).

2. Pui, C.H., Relling, M.V. \& Downing, J.R. Acute lymphoblastic leukemia. N. Engl. J. Med. 350, 1535-1548 (2004)

3. Paietta, E. et al. Activating FLT3 mutations in CD117/KIT positiveT-cell acute lymphoblastic leukemias. Blood 104, 558-560 (2004).

4. Maurer, B.J., Lai, E., Hamkalo, B.A., Hood, L. \& Attardi, G. Novel submicroscopic extrachromosomal elements containing amplified genes in human cells. Nature 327, 434-437 (1987).

5. de Klein, A. et al. A cellular oncogene is translocated to the Philadelphia chromosome in chronic myelocytic leukaemia. Nature 300, 765-767 (1982).

6. von Lindern, M. et al. The translocation (6;9), associated with a specific subtype of acute myeloid leukemia, results in the fusion of two genes, dek and can, and the expression of a chimeric, leukemia-specific dek-can mRNA. Mol. Cell Biol. 12, 1687 1697 (1992).

7. Capdeville, R., Buchdunger, E., Zimmermann, J. \& Matter, A. Glivec (STI571, imatinib), a rationally developed, targeted anticancer drug. Nat. Rev. Drug Discov. 1, 493-502 (2002).

8. Druker, B.J. et al. Activity of a specific inhibitor of the BCR-ABL tyrosine kinase in the blast crisis of chronic myeloid leukemia and acute lymphoblastic leukemia with the Philadelphia chromosome. N. Engl. J. Med. 344, 1038-1042 (2001).

9. Hebert, J., Cayuela, J.M., Berkeley, J. \& Sigaux, F. Candidate tumor-suppressor genes MTS1 (p16INK4A) and MTS2 (p15INK4B) display frequent homozygous deletions in primary cells from T- but not from B-cell lineage acute lymphoblastic leukemias. Blood 84, 4038-4044 (1994).

10. de Klein, A. et al. bcr rearrangement and translocation of the c-abl oncogene in Philadelphia positive acute lymphoblastic leukemia. Blood 68, 1369-1375 (1986).

11. Hahn, P.J. Molecular biology of double-minute chromosomes. Bioessays 15, 477-484 (1993).

12. Carroll, S.M. et al. Double minute chromosomes can be produced from precursors derived from a chromosomal deletion. Mol. Cell Biol. 8, 1525-1533 (1988).

13. Kraemer, D., Wozniak, R.W., Blobel, G. \& Radu, A. The human CAN protein, a putative oncogene product associated with myeloid leukemogenesis, is a nuclear pore complex protein that faces the cytoplasm. Proc. Natl. Acad. Sci. USA 91, 1519-1523 (1994).

14. Golub, T.R. et al. Oligomerization of the ABL tyrosine kinase by the Ets protein TEL in human leukemia. Mol. Cell Biol. 16, 4107-4116 (1996).

15. Oda, T. et al. Crkl is the major tyrosine-phosphorylated protein in neutrophils from patients with chronic myelogenous leukemia. J. Biol. Chem. 269, 22925-22928 (1994).

16. Nieborowska-Skorska, M., Slupianek, A. \& Skorski, T. Progressive changes in the leukemogenic signaling in BCR/ABL-transformed cells. Oncogene 19, 4117-4124 (2000).

17. Kelly, L.M. \& Gilliland, D.G. Genetics of myeloid leukemias. Annu. Rev. Genomics Hum. Genet. 3, 179-198 (2002).

18. Nagel, S., Kaufmann, M., Drexler, H.G. \& MacLeod, R.A. The cardiac homeobox gene NKX2-5 is deregulated by juxtaposition with BCL11B in pediatric T-ALL cell lines via a novel $\mathrm{t}(5 ; 14)(\mathrm{q} 35.1 ; \mathrm{q} 32.2)$. Cancer Res. 63, 5329-5334 (2003).

19. Barber, K.E. et al. Amplification of the ABL gene in T-cell acute lymphoblastic leukemia. Leukemia 18, 1153-1156 (2004).

20. Cools, J. et al. A tyrosine kinase created by fusion of the PDGFRA and FIP1L1 genes as a therapeutic target of imatinib in idiopathic hypereosinophilic syndrome. N. Engl. J. Med. 348, 1201-1214 (2003).

21. Mitelman, F. ISCN(1995): Guidelines for Cancer Cytogenetics. Supplement to an International System for Human Nomenclature (Karger, Basel, 1995).

22. Dierlamm, J. et al. Successful use of the same slide for consecutive fluorescence in situ hybridization experiments. Genes Chromosomes Cancer 16, 261-264 (1996).

23. Fiegler, $\mathrm{H}$. et al. DNA microarrays for comparative genomic hybridization based on DOP-PCR amplification of BAC and PAC clones. Genes Chromosomes Cancer 36, 361374 (2003).

24. Van Buggenhout, G. et al. The mild Wolf-Hirschhorn syndrome: microarray CGH analysis of atypical 4 p16.3 deletions enables refinement of the genotype-phenotype map. J. Med. Genet. 41, 691-698 (2004).

25. Fornerod, M. et al. Relocation of the carboxyterminal part of CAN from the nuclear envelope to the nucleus as a result of leukemia-specific chromosome rearrangements. Oncogene 10, 1739-1748 (1995).

26. Cools, J. et al. Fusion of a novel gene, BTL, to ETV6 in acute myeloid leukemias with a $\mathrm{t}(4 ; 12)$ (q11-q12;p13). Blood 94, 1820-1824 (1999).

27. Bene, M.C. et al. Proposals for the immunological classification of acute leukemias. European Group for the Immunological Characterization of Leukemias (EGIL). Leukemia 9, 1783-1786 (1995). 